\title{
New existence of hyperbolic orbits for a class of singular Hamiltonian systems
}

Dong-Lun $\mathrm{Wu}^{*}$ and Shiqing Zhang

\section{${ }^{\text {"Correspondence: }}$} wud12008@163.com Yangtze Center of Mathematics and College of Mathematics, Sichuan University, Chengdu, 610064, People's Republic of China

\begin{abstract}
A new existence of hyperbolic orbits is obtained for a class of singular Hamiltonian systems with prescribed energies by taking the limit for a sequence of approximate solutions. Furthermore, we show that the hyperbolic orbits possess the given directions at infinity.
\end{abstract}

Keywords: hyperbolic orbits; variational methods; prescribed energy; singular Hamiltonian systems; asymptotic directions

\section{Introduction and main results}

In this paper, we consider the following Hamiltonian systems with prescribed energy:

$$
\ddot{u}(t)+\nabla V(u(t))=0
$$

with

$$
\frac{1}{2}|\dot{u}(t)|^{2}+V(u(t))=H,
$$

where $u \in C^{2}\left(R, R^{N}\right), V \in C^{1}\left(R^{N} \backslash\{0\}, R\right)$ has a singularity at the origin. $\nabla V(x)$ denotes the gradient with respect to the $x$ variable, $(\cdot, \cdot): R^{N} \times R^{N} \rightarrow R$ denotes the standard Euclidean inner product in $R^{N}$ and $|\cdot|$ is the induced norm. The parabolic and hyperbolic orbits are defined as follows.

Definition 1 (see [1]) If $u(t)$ is a solution for problem (1.1)-(1.2) satisfying

$$
|u(t)| \rightarrow+\infty \text { and }|\dot{u}(t)| \rightarrow 0 \quad \text { as } t \rightarrow \pm \infty,
$$

then $u(t)$ is called a parabolic orbit.

If $u(t)$ satisfies

$$
|u(t)| \rightarrow+\infty \text { and }|\dot{u}(t)|>0 \quad \text { as } t \rightarrow \pm \infty,
$$

then $u(t)$ is called a hyperbolic orbit.

(c) 2015 Wu and Zhang. This article is distributed under the terms of the Creative Commons Attribution 4.0 International License (http://creativecommons.org/licenses/by/4.0/), which permits unrestricted use, distribution, and reproduction in any medium, provided you give appropriate credit to the original author(s) and the source, provide a link to the Creative Commons license, and indicate if changes were made. 
The solutions of Hamiltonian systems have been studied by many mathematicians (see [1-18] and the references therein). In 1922, Chazy showed that there are only seven possible final evolutions in the three-body problem. And the parabolic and hyperbolic orbits have been obtained for problem (1.1) when $V$ is singular at the origin by $[4,9,19]$ with variational methods. In [19], the authors obtained the existence of collision-free parabolic orbits for a Newtonian $n$-body problem starting from any initial configuration and asymptotic to every minimizing normalized central configuration.

In this paper, we mainly consider the strong force case. For this case, in 2000, Felmer and Tanaka obtained the following theorem.

Theorem A (see [9]) Assume that $N \geq 3$ and the following conditions hold:

$\left(\mathrm{A}_{1}\right) V \in C^{1}\left(R^{N} \backslash\{0\}, R\right)$,

$\left(\mathrm{A}_{2}\right) \quad V(x) \leq 0$ for all $x \in R^{N} \backslash\{0\}$,

$\left(\mathrm{A}_{3}\right)$ there are constants $\zeta>2, \rho>0$ and $d_{0}>0$ such that

(i) $-V(x) \geq \frac{d_{0}}{|x| 5}$ for $0<|x| \leq \rho$,

(ii) $(x, \nabla V(x))+2 V(x) \rightarrow+\infty$ as $|x| \rightarrow 0$,

$\left(\mathrm{A}_{4}\right)$ there exist $v>2$ and $C>0$ such that

$$
-V(x) \leq \frac{C}{|x|^{v-1}} \quad \text { and } \quad|\nabla V(x)| \leq \frac{C}{|x|^{v}} \quad \text { for }|x| \geq 1
$$

Then, for any given $H>0$ and $\theta_{+} \neq-\theta_{-}$, there exists a solution $u(t)$ of (1.1)-(1.2) such that

$$
\lim _{t \rightarrow \pm \infty} \frac{u(t)}{|u(t)|}=\theta_{ \pm}
$$

where $\theta_{+}, \theta_{-} \in S^{N-1}=\left\{x \in R^{N}|| x \mid=1\right\}$ are the asymptotic directions for the solution $u(t)$.

The proof of Theorem A depends on the difference of the given asymptotic directions. In this paper, we try to relax the conditions on the asymptotic directions and the growth condition $\left(\mathrm{V}_{4}\right)$. First, we consider problem (1.1)-(1.2) on a bounded interval, and then we let the interval go to infinity to get hyperbolic orbits. The following theorems are our main results.

Theorem 1 Suppose $N \geq 3$ and $V \in C^{1}\left(R^{N} \backslash\{0\}, R\right)$ satisfies $\left(\mathrm{A}_{1}\right),\left(\mathrm{A}_{2}\right)$ and the following conditions:

$\left(\mathrm{V}_{1}\right) 2 V(x)+(x, \nabla V(x)) \rightarrow+\infty$ as $|x| \rightarrow 0$,

$\left(\mathrm{V}_{2}\right) \quad V(x) \rightarrow-\infty$ as $|x| \rightarrow 0$,

$\left(\mathrm{V}_{3}\right)(\nabla V(x), x) \rightarrow 0$ as $|x| \rightarrow+\infty$,

$\left(\mathrm{V}_{4}\right)$ there exist constants $\beta>2, M_{0}>0$ and $\sigma_{1} \geq 1$ such that

$$
|x|^{\beta}|V(x)| \leq M_{0} \quad \text { for all }|x| \geq \sigma_{1}
$$

Then, for any $H>0$, there exists at least one hyperbolic orbit for problem (1.1)-(1.2). 
Remark 1 Compared with condition $\left(\mathrm{A}_{4}\right)$, there is no condition on $\nabla V(x)$ in $\left(\mathrm{V}_{4}\right)$, and we can give an example which satisfies $\left(\mathrm{V}_{4}\right)$, but not condition $\left(\mathrm{A}_{4}\right)$, such as

$$
V(x)= \begin{cases}-\frac{\ln \left(\left.\left|x x^{2}+\right| x\right|^{-2}\right)}{|x|^{2}} & \text { for }|x| \leq 1, \\ Q(x) & \text { for } 1 \leq|x| \leq 2, \\ -\frac{2+\sin |x|^{2}}{|x|^{3}} & \text { for }|x| \geq 2,\end{cases}
$$

where $Q(x) \in C^{1}\left(R^{N}, R\right)$ such that $V(x)(\leq 0)$ is of $C^{1}$ class. It is easy to see that (1.3) also satisfies $\left(\mathrm{A}_{1}\right),\left(\mathrm{A}_{2}\right),\left(\mathrm{V}_{1}\right),\left(\mathrm{V}_{2}\right)$ and $\left(\mathrm{V}_{3}\right)$.

In order to estimate the asymptotic direction of the hyperbolic orbit, we need to strengthen condition $\left(\mathrm{V}_{3}\right)$, which is the following theorem.

Theorem 2 Suppose $N \geq 3$ and $V \in C^{1}\left(R^{N} \backslash\{0\}, R\right)$ satisfies $\left(\mathrm{A}_{1}\right),\left(\mathrm{A}_{2}\right),\left(\mathrm{V}_{1}\right),\left(\mathrm{V}_{2}\right),\left(\mathrm{V}_{4}\right)$ and the following condition:

$\left(\mathrm{V}_{5}\right)$ there exist $\kappa>2, \rho_{0}>0$ and $\sigma_{2}>0$ such that

$$
|x|^{\kappa}|\nabla V(x)| \leq \rho_{0} \quad \text { for all }|x| \geq \sigma_{2} .
$$

Then, for any $H>0$, there exists at least one hyperbolic orbit for problem (1.1)-(1.2) which possesses any given asymptotic directions at infinity.

Remark 2 There is no restriction on the asymptotic directions of the hyperbolic solution at infinity in Theorems 1 and 2, which is different from Theorem A, and the restriction on the asymptotic directions is important in the proof of the blow-up argument.

Remark 3 Since the total energy is a positive constant, to show a solution $u(t)$ is a hyperbolic solution, we just need to show that $|u(t)| \rightarrow \infty$ as $t \rightarrow \pm \infty$.

The paper is organized as follows. In Section 2, we present some preliminaries. In Section 3, we obtain the existence of approximate solutions. In Section 4, we give some estimates of the approximate solutions. In Section 5, we give the proof of Theorem 1. In Section 6, we give the proof of Theorem 2 .

\section{Variational settings}

For any given two unite vectors (directions) $e_{ \pm} \in S^{N-1}$, we set

$$
\begin{aligned}
& H^{1}=W^{1,2}\left(R / Z, R^{N}\right), \\
& \Lambda_{r}=\left\{q \in H^{1} \mid q(0)=r e_{-}, q(1)=r e_{+}, q \neq 0 \text { for all } t \in[0,1]\right\},
\end{aligned}
$$

with the norm

$$
\|q\|:=\left(\int_{0}^{1}|\dot{q}(t)|^{2} d t\right)^{1 / 2}+|q(0)|
$$

Here we use $r$ to denote the Euclidean length of $q(0)$ and $q(1)$. Furthermore, for convenience, we let $r>1$ in the following proof. Let $L^{\infty}\left([0,1], R^{N}\right)$ be a space of measurable 
functions from $[0,1]$ into $R^{N}$ and essentially bounded with the following norm:

$$
\|q\|_{L^{\infty}\left([0,1], R^{N}\right)}:=\operatorname{esssup}\{|q(t)|: t \in[0,1]\} .
$$

Moreover, let $f: \Lambda_{r} \rightarrow R$ be the functional defined by

$$
f(q)=\frac{1}{2} \int_{0}^{1}|\dot{q}(t)|^{2} d t \int_{0}^{1}(H-V(q(t))) d t .
$$

Then one can easily check that $f \in C^{1}\left(\Lambda_{r}, R\right)$ and

$$
\begin{aligned}
\left\langle f^{\prime}(q), p\right\rangle= & \int_{0}^{1}\langle\dot{q}(t), \dot{p}(t)\rangle d t \int_{0}^{1}(H-V(q(t))) d t \\
& -\frac{1}{2} \int_{0}^{1}|\dot{q}(t)|^{2} d t \int_{0}^{1}(\nabla V(q(t)), p(t)) d t .
\end{aligned}
$$

Our way to get the hyperbolic orbit is to approach it with a sequence of approximate solutions. Firstly, we prove the existence of the approximate solutions, then we study the limit procedure. We consider the following approximate problems:

$$
\ddot{u}(t)+\nabla V(u(t))=0, \quad \forall t \in\left(-T_{r}, T_{r}\right)
$$

with

$$
\begin{aligned}
& \frac{1}{2}|\dot{u}(t)|^{2}+V(u(t))=H, \quad \forall t \in\left(-T_{r}, T_{r}\right), \\
& u\left(-T_{r}\right)=r e_{-}, \quad u\left(T_{r}\right)=r e_{+},
\end{aligned}
$$

where $T_{r}$ is a suitable number depending on the critical points of $f$ and $r$ which will be given in the following lemma.

\section{Existence of approximate solutions}

The approximate solutions are obtained by the minimax methods. It is known that the critical points of $f$ correspond to the approximate solutions after an appropriate scaling of time. The following lemma shows this fact.

Lemma 3.1 (see [1]) Let $f(q)=\frac{1}{2} \int_{0}^{1}|\dot{q}(t)|^{2} d t \int_{0}^{1}(H-V(q(t))) d t$ and $\tilde{q} \in \Lambda_{r}$ be such that $f^{\prime}(\tilde{q})=0, f(\tilde{q})>0$. Set

$$
T^{2}=\frac{\frac{1}{2} \int_{0}^{1}|\dot{\tilde{q}}(t)|^{2} d t}{\int_{0}^{1}(H-V(\tilde{q}(t))) d t} .
$$

Then $\tilde{u}(t)=\tilde{q}(t / T)$ is a non-constant $T$-periodic solution for problem (1.1)-(1.2).

In this paper, we need to let the time $t$ tend to $\pm \infty$. So when we scale the time, we translate $t$ to a suitable interval so that the function is still a solution after the translation, which can be guaranteed by the following lemma. 
Lemma 3.2 (Translation property [13]) Suppose that in the domain $D \subset R^{N}$, we have a solution $\phi(t)$ for the following differential equation:

$$
x^{(n)}+F\left(x^{(n-1)}, \ldots, x\right)=0,
$$

where $x^{(k)}=d^{k} x / d t^{k}, k=0,1, \ldots, n, x^{(0)}=x$. Then $\phi\left(t-t_{0}\right)$ with $t_{0}$ being a constant is also a solution.

Next, we introduce Gordon's strong force condition.

Lemma 3.3 (Strong force condition [10]) $V$ is said to satisfy Gordon's strongforce condition if there exist a neighborhood $\mathcal{N}$ of 0 and a function $U \in C^{1}\left(R^{N} \backslash\{0\}, R\right)$ such that

(i) $\lim _{x \rightarrow 0} U(x)=-\infty$;

(ii) $-V(x) \geq\left|U^{\prime}(x)\right|^{2}$ for every $x \in \mathcal{N} \backslash\{0\}$.

It has been shown that if $V$ satisfies Gordon's strong force condition, then

$$
\int_{0}^{1} V\left(x_{j}\right) d t \rightarrow-\infty, \quad \forall x_{j} \rightarrow x \in \partial \Lambda_{r}
$$

Lemma 3.4 Suppose $\left(\mathrm{A}_{2}\right),\left(\mathrm{V}_{1}\right)$ and $\left(\mathrm{V}_{2}\right)$ hold, then $V$ satisfies Gordon's strong force condition.

Proof Let $\phi(r)=-V(\tilde{e}) r^{2}$, where $r=|x|, \tilde{e}=x /|x|$, then we have

$$
\phi^{\prime}(r)=-r(2 V(\widetilde{r e})+(\nabla V(r \widetilde{e}), \widetilde{r e})) .
$$

It follows from $\left(\mathrm{V}_{1}\right)$ and $\left(\mathrm{V}_{2}\right)$ that there exists a constant $\delta>0$ such that

$$
\phi^{\prime}(r) \leq 0 \quad \text { and } \quad \min _{|x|=\delta}(-V(x))>0 \quad \text { for all } 0<r \leq \delta
$$

Then we get

$$
-V(r \widetilde{e}) r^{2}=\phi(r) \geq \phi(\delta)=-V(\delta \tilde{e}) \delta^{2} \geq \delta^{2} \min _{|x|=\delta}(-V(x)) .
$$

It follows from the definition of $\phi$ and $\left(\mathrm{V}_{2}\right)$ that there exists a constant $C_{1}>0$ such that

$$
-V(x) \geq \frac{C_{1}}{|x|^{2}} \quad \text { for all } 0<|x| \leq \delta
$$

We set $U(x)=\sqrt{C_{1}} \ln |x|$, then by an easy calculation we obtain

$$
\lim _{x \rightarrow 0} U(x)=-\infty \quad \text { and } \quad-V(x) \geq\left|U^{\prime}(x)\right|^{2} \quad \text { for all } 0<r \leq \delta,
$$

which proves this lemma.

Subsequently, we look for the minimax type critical points of $f$. 
Lemma 3.5 Suppose that the conditions of Theorem 1 hold, then for any $r>1$ there exists at least one approximate solution on $\Lambda_{r}$ for problem (2.1)-(2.3), where $T_{r}$ is defined in the proof.

Proof We set

$$
\Omega_{r}=\left\{\gamma \in C\left(S^{N-2}, \Lambda_{r}\right) \mid \operatorname{deg}(\tilde{\gamma})=1\right\}
$$

where

$$
\tilde{\gamma}(\xi, t)=\frac{\gamma(\xi)(t)}{|\gamma(\xi)(t)|}: S^{N-2} \times(R / Z) \simeq S^{N-2} \times S^{1} \rightarrow S^{N-1},
$$

and $\operatorname{deg}(\tilde{\gamma})$ denotes the Brouwer degree of $\tilde{\gamma}$. We show that $f$ satisfies the $(P S)^{+}$condition on $\Lambda_{r}$. Specifically, let $c>0,\left\{q_{j}\right\} \subset \Lambda_{r}$ such that $f\left(q_{j}\right) \rightarrow c$ and $f^{\prime}\left(q_{j}\right) \rightarrow 0$. By the definition of $f, H>0$ and $V \leq 0$, we can deduce that $\left\|\dot{q}_{j}\right\|_{L^{2}}$ is bounded. Then there exists a constant $C_{0}>0$ such that

$$
\left\|q_{j}-\left[q_{j}\right]\right\|_{L^{\infty}} \leq C_{0}\left\|\dot{q}_{j}\right\|_{L^{2}}
$$

where $\left[q_{j}\right]=\int_{0}^{1} q_{j}(t) d t$. If $\left[q_{j}\right]$ is unbounded, we obtain that $\left\|q_{j}\right\|_{L^{\infty}}$ is also unbounded. It follows from $\left(\mathrm{V}_{4}\right)$ and (3.2) that

$$
\int_{0}^{1}\left(\nabla V\left(q_{j}(t)\right), q_{j}(t)-\left[q_{j}\right]\right) d t \rightarrow 0 \quad \text { as } j \rightarrow+\infty
$$

Then it is easy to see that

$$
2 f\left(q_{j}\right)=f^{\prime}\left(q_{j}\right)\left(q_{j}(t)-\left[q_{j}\right]\right)+\frac{1}{2}\left\|\dot{q}_{j}\right\|_{L^{2}}^{2} \int_{0}^{1}\left(\nabla V\left(q_{j}(t)\right), q_{j}(t)-\left[q_{j}\right]\right) d t \rightarrow 0
$$

as $j \rightarrow+\infty$, which is a contradiction. Then we can deduce that $\left[q_{j}\right]$ is bounded. Together with the boundedness of $\left\|\dot{q}_{j}\right\|_{L^{2}}$, we obtain that $q_{j}$ is bounded in $\Lambda_{r}$, which implies that there exists a subsequence of $\left\{q_{j}\right\}$, still denoted by $\left\{q_{j}\right\}$, such that $q_{j} \rightarrow q$. Moreover, $q_{j} \rightarrow q$ uniformly on $[0,1]$. It follows from Lemmas 3.3 and 3.4 that $q \notin \partial \Omega_{r}=\left\{q \in E_{R} \mid \exists t^{\prime} \in\right.$ $[0,1]$ s.t. $\left.q\left(t^{\prime}\right)=0\right\}$. Otherwise, if $q$ has collision, which means $q \in \partial \Omega_{r}$, we can prove that

$$
f\left(q_{j}\right) \rightarrow+\infty \text { as } j \rightarrow+\infty .
$$

To prove this fact, there are two cases needed to be discussed.

Case 1. If $q=$ constant, it follows from $q \in \partial \Omega_{r}$ that $q \equiv 0$, which is a contradiction since $|q(0)|=|q(1)|=r$.

Case 2. If $q \neq$ constant, we have $\int_{0}^{1}|\dot{q}(t)|^{2} d t>0$. Then by the weakly-lower-semicontinuity of norm, we have

$$
\liminf _{j \rightarrow \infty}\left\|q_{j}\right\|=\liminf _{j \rightarrow \infty}\left(\int_{0}^{1}\left|\dot{q}_{j}(t)\right|^{2} d t\right)^{1 / 2}+r \geq\|q\|=\left(\int_{0}^{1}|\dot{q}(t)|^{2} d t\right)^{1 / 2}+r
$$


which implies that

$$
\liminf _{n \rightarrow \infty} \int_{0}^{1}\left|\dot{q}_{j}(t)\right|^{2} d t>0
$$

By Lemmas 3.3, 3.4 and (3.1), we can deduce that (3.5) holds, which is a contradiction. Then we can see that $q \in \Omega_{r}$ has no collision. By a standard argument, we can see $q_{j} \rightarrow q$ in $\Omega_{r}$, which proves the $(P S)^{+}$condition.

Then the minimax value is introduced by

$$
b_{r}=\inf _{\gamma \in \Omega_{r}} \max _{\xi \in S^{N-2}} f(\gamma(\xi)) .
$$

It follows from $f(q) \geq 0$ that $b_{r} \geq 0$. But it follows from the definition of $f$ that the necessary condition for $b_{r}=0$ is $\gamma(\xi) \equiv$ constant, which contradicts with the definition of $\Omega_{r}$. Via the standard minimax argument (similar to [5]), we can see that $b_{r}$ is a critical value of $f$ and there exists $q_{r} \in \Omega_{r}$ such that

$$
f\left(q_{r}\right)=b_{r}>0, \quad f^{\prime}\left(q_{r}\right)=0 .
$$

Let

$$
T_{r}^{2}=\frac{\frac{1}{2} \int_{0}^{1}\left|\dot{q}_{r}(t)\right|^{2} d t}{\int_{0}^{1}\left(H-V\left(q_{r}(t)\right)\right) d t} .
$$

Then, by Lemmas 3.1 and 3.2, we obtain that $u_{r}(t)=q_{r}\left(\frac{t+T_{r}}{2 T_{r}}\right):\left(-T_{r}, T_{r}\right) \rightarrow H^{1}$ is a nontrivial solution for problem (2.1)-(2.3). The lemma is proved.

\section{Blowing-up argument}

In order to process the limit procedure, it is necessary to show that the minimum of $\left|u_{r}(t)\right|$ has a uniform bound from above which guarantees that the asymptotic solutions cannot diverge to infinity as $r \rightarrow+\infty$. Specifically, we obtain the following lemma.

Lemma 4.1 Suppose that $u_{r}(t):\left(-T_{r}, T_{r}\right) \rightarrow H^{1}$ is the solution obtained in Lemma 3.5, then $\min _{t \in\left(-T_{r}, T_{r}\right)}\left|u_{r}(t)\right|$ is bounded from above uniformly. Specifically, there is a constant $M>0$ independent of $r$ such that

$$
\min _{t \in\left(-T_{r}, T_{r}\right)}\left|u_{r}(t)\right| \leq M \quad \text { for all } r>1
$$

Proof Since $f^{\prime}\left(q_{r}\right)=0$ and $q_{r} \not \equiv 0$, we can obtain that $\left\langle f^{\prime}\left(q_{r}\right), q_{r}\right\rangle=0$, which implies that

$$
\int_{0}^{1} 2 H-2 V\left(q_{r}(t)\right)-\left(\nabla V\left(q_{r}(t)\right), q_{r}(t)\right) d t=0
$$

Then we obtain that

$$
\int_{-T_{r}}^{T_{r}} 2 H-2 V\left(u_{r}(t)\right)-\left(\nabla V\left(u_{r}(t)\right), u_{r}(t)\right) d t=0 .
$$


It can be convinced that there exists $\hat{t} \in\left[-T_{r}, T_{r}\right]$ such that

$$
2 H-2 V\left(u_{r}(\hat{t})\right)-\left(\nabla V\left(u_{r}(\hat{t})\right), u_{r}(\hat{t})\right) \leq 0
$$

which implies that

$$
2 H \leq 2 V\left(u_{r}(\hat{t})\right)+\left(\nabla V\left(u_{r}(\hat{t})\right), u_{r}(\hat{t})\right) .
$$

It follows from $\left(\mathrm{V}_{3}\right)$ and $\left(\mathrm{V}_{4}\right)$ that there exists a constant $M_{1}>0$ independent of $r$ such that

$$
\min _{t \in\left(-T_{r}, T_{r}\right)}\left|u_{r}(t)\right| \leq M_{1}
$$

Then we finish the proof of this lemma.

\section{Proof of Theorem 1}

In this section, we prove the existence of hyperbolic orbits by some estimates of asymptotic solutions. Firstly, we prove that the asymptotic solutions are uniformly collision-free, which can be shown by the strong force condition.

Lemma 5.1 Suppose that $u_{r}(t)$ is the solution for (2.1)-(2.3) obtained in Lemma 3.5. Then there exists a constant $m>0$ independent of $r$ such that

$$
\min _{t \in\left(-T_{r}, T_{r}\right)}\left|u_{r}(t)\right| \geq m
$$

Proof Since $u_{r}(t)$ is a solution for problem (2.1)-(2.3), then we can deduce that

$$
\begin{aligned}
\frac{d^{2}}{d t^{2}} \frac{1}{2}\left|u_{r}(t)\right|^{2} & =\frac{d}{d t}\left(u_{r}(t), \dot{u}_{r}(t)\right) \\
& =\left|\dot{u}_{r}(t)\right|^{2}+\left(u_{r}(t), \ddot{u}_{r}(t)\right) \\
& =2\left(H-V\left(u_{r}(t)\right)\right)-\left(\nabla V\left(u_{r}(t)\right), u_{r}(t)\right), \quad t \in\left(-T_{r}, T_{r}\right) .
\end{aligned}
$$

By hypothesis $\left(\mathrm{V}_{1}\right)$, we can find $m \in(0,1)$ independent of $r$ such that

$$
2 H-\left(2 V\left(u_{r}(t)\right)+\left(\nabla V\left(u_{r}(t)\right), u_{r}(t)\right)\right)<0
$$

for any $t \in\left\{t \in\left(-T_{r}, T_{r}\right)\left|\max _{t \in\left(-T_{r}, T_{r}\right)}\right| u_{r}(t) \mid \leq m\right\}$, which implies that $\left|u_{r}(t)\right|$ is concave when $\left|u_{r}(t)\right| \leq m$ and $\left|u_{r}(t)\right|$ cannot take a local minimum such that $\max _{t \in\left(-T_{r}, T_{r}\right)}\left|u_{r}(t)\right| \leq$ $m$, which implies that

$$
\left|u_{r}(t)\right| \geq m \text { for all } t \in\left(-T_{r}, T_{r}\right) .
$$

If not, we can assume that there exists $\bar{t} \in\left(-T_{r}, T_{r}\right)$ such that $\left|u_{r}(\bar{t})\right|<m$, then we can easily check that $\left|u_{r}(t)\right|$ takes a local minimum at some $\tilde{t}$ with $\left|u_{r}(\tilde{t})\right|<m$, which is a contradiction. Then we obtain the conclusion. 
The proofs of the following two lemmas are similar to those in [9] and [18], we sketch the proofs for the reader's convenience.

Lemma 5.2 Suppose that $r>\max \left\{M, \sigma_{1}, 1\right\}$, where $M$ is defined in Lemma 4.1 and $u_{r}(t)$ is the solution for problem (2.1)-(2.3) obtained in Lemma 3.5. Set

$$
t_{+}=\sup \left\{t \in\left[-T_{r}, T_{r}\right]|| u_{r}(t) \mid \leq L\right\}
$$

and

$$
t_{-}=\inf \left\{t \in\left[-T_{r}, T_{r}\right]|| u_{r}(t) \mid \leq L\right\},
$$

where $L$ is a constant independent of $r$ such that $M<L<r$. Then we have that

$$
T_{r}-t_{+} \rightarrow+\infty, \quad t_{-}+T_{r} \rightarrow+\infty \quad \text { as } r \rightarrow+\infty
$$

Proof By the definition of $u_{r}(t)$ we have

$$
\left|u_{r}\left(-T_{r}\right)\right|=\left|u_{r}\left(T_{r}\right)\right|=r .
$$

Then, by $\left(\mathrm{A}_{2}\right)$ and the definitions of $t_{+}$and $t_{-}$, we obtain

$$
\begin{aligned}
\int_{t_{+}}^{T_{r}} \sqrt{H-V\left(u_{r}(t)\right)}\left|\dot{u}_{r}(t)\right| d t & \geq \sqrt{H} \int_{t_{+}}^{T_{r}}\left|\dot{u}_{r}(t)\right| d t \\
& \geq \sqrt{H}\left|\int_{t_{+}}^{T_{r}} \dot{u}_{r}(t) d t\right| \geq \sqrt{H}(r-L)
\end{aligned}
$$

and

$$
\begin{aligned}
\int_{-T_{r}}^{t_{-}} \sqrt{H-V\left(u_{r}(t)\right)}\left|\dot{u}_{r}(t)\right| d t & \geq \sqrt{H} \int_{-T_{r}}^{t_{-}}\left|\dot{u}_{r}(t)\right| d t \\
& \geq \sqrt{H}\left|\int_{-T_{r}}^{t_{-}} \dot{u}_{r}(t) d t\right| \geq \sqrt{H}(r-L) .
\end{aligned}
$$

Since $V \in C^{1}\left(R^{N} \backslash\{0\}, R\right)$, it follows from Lemma 5.1 and $\left(\mathrm{V}_{4}\right)$ that there exists a constant $M_{2}>0$ independent of $r$ such that

$$
\left|V\left(u_{r}(t)\right)\right| \leq M_{2} \quad \text { for all } t \in\left[-T_{r}, T_{r}\right]
$$

which implies that

$$
\int_{t_{+}}^{T_{r}} \sqrt{H-V\left(u_{r}(t)\right)}\left|\dot{u}_{r}(t)\right| d t=\sqrt{2} \int_{t_{+}}^{T_{r}}\left(H-V\left(u_{r}(t)\right)\right) d t \leq \sqrt{2}\left(H+M_{2}\right)\left(T_{r}-t_{+}\right) .
$$

Combining (5.1) with the above inequality, we obtain that

$$
\sqrt{H}(r-L) \leq \sqrt{2}\left(H+M_{2}\right)\left(T_{r}-t_{+}\right)
$$


Then we have

$$
T_{r}-t_{+} \rightarrow+\infty \quad \text { as } r \rightarrow+\infty
$$

The limit for $t_{-}+T_{r}$ can be obtained in a similar way. The proof is completed.

Lemma 5.3 Suppose that $u_{r}(t)$ is the solution for problem (2.1)-(2.3) obtained in Lemma 3.5. Then there exists a constant $M_{3}>0$ independent of $r>1$ such that

$$
\int_{-T_{r}}^{T_{r}} \sqrt{H-V\left(u_{r}(t)\right)}\left|\dot{u}_{r}(t)\right| d t \leq 2 \sqrt{H} r+M_{3}
$$

Proof Firstly, we define the function $\xi(t)$ on $[1,+\infty)$ as a solution of

$$
\begin{aligned}
& \dot{\xi}(t)=\sqrt{2\left(H-V\left(\xi(t) e_{0}\right)\right)}, \\
& \xi(1)=\sigma_{1},
\end{aligned}
$$

where $\sigma_{1}$ is defined in $\left(\mathrm{V}_{4}\right)$ and $e_{0} \in S^{N-1}$. And $\tau_{r}>1$ is a real number such that $\xi\left(\tau_{r}\right)=r$. We can define $\xi(t)$ in $(-\infty, 0]$ and $\tau_{-r}$ in a similar way. Then we can fix $\varphi(t) \in \Omega_{1}$ (defined in Lemma 3.5) such that $\tilde{\gamma}_{r}(t) \in \Omega_{r}$, where

$$
\tilde{\gamma}_{r}(t)=\gamma_{r}\left(t\left(\tau_{r}-\tau_{-r}\right)+\tau_{-r}\right) \quad \text { and } \quad \gamma_{r}(t)= \begin{cases}\xi(t) e_{0} & \text { for } t \in\left[1, \tau_{r}\right] \cup\left[\tau_{-r}, 0\right] \\ \varphi(t) & \text { for } t \in[0,1]\end{cases}
$$

Subsequently, we set $u_{r}(t)=\tilde{\gamma}_{r}\left(\frac{t+a}{2 a}\right)$ for any $a>0$, then it is easy to see that $u_{r}(t)=\gamma_{r}(t)$ if $\tau_{ \pm r}= \pm a$. Similar to [9], we can deduce that for $a>0$

$$
\begin{aligned}
\left(2 f\left(\tilde{\gamma}_{r}\right)\right)^{\frac{1}{2}} & =\inf _{a>0} \frac{1}{\sqrt{2}} \int_{-a}^{a} \frac{1}{2}\left|\dot{u}_{r}(t)\right|^{2}+H-V\left(u_{r}(t)\right) d t \\
& \leq \frac{1}{\sqrt{2}} \int_{-\tau_{r}}^{\tau_{r}} \frac{1}{2}\left|\dot{\gamma}_{r}(t)\right|^{2}+H-V\left(\gamma_{r}(t)\right) d t \\
& =I_{\left[-\tau_{r}, \tau_{r}\right]} .
\end{aligned}
$$

We divide the interval $\left[-\tau_{r}, \tau_{r}\right]$ into three parts $\left[-\tau_{r}, 0\right] \cup[0,1] \cup\left[1, \tau_{r}\right]$, then we can estimate $I_{\left[-\tau_{r}, \tau_{r}\right]}$ by three integrals. Firstly, we estimate $I_{\left[1, \tau_{r}\right]}$. By $\left(\mathrm{V}_{4}\right)$, we have

$$
\begin{aligned}
I_{\left[1, \tau_{r}\right]} & =\frac{1}{\sqrt{2}} \int_{1}^{\tau_{r}} \frac{1}{2}\left|\dot{\gamma}_{r}(t)\right|^{2}+H-V\left(\gamma_{r}(t)\right) d t \\
& =\int_{1}^{\tau_{r}} \sqrt{H-V\left(\xi(t) e_{0}\right)} \dot{\xi}(t) d t \\
& =\int_{\sigma_{1}}^{r} \sqrt{H-V\left(s e_{0}\right)} d s \\
& \leq \int_{\sigma_{1}}^{r} \sqrt{H}+\sqrt{-V\left(s e_{0}\right)} d s \\
& =\sqrt{H}\left(r-\sigma_{1}\right)+\int_{\sigma_{1}}^{r} \sqrt{-V\left(s e_{0}\right)} d s
\end{aligned}
$$




$$
\begin{aligned}
& \leq \sqrt{H} r+\sqrt{M_{0}} \int_{\sigma_{1}}^{r} s^{-\frac{\beta}{2}} d s \\
& \leq \sqrt{H} r+\sqrt{M_{0}} \int_{\sigma_{1}}^{+\infty} s^{-\frac{\beta}{2}} d s \\
& \leq \sqrt{H} r+M_{4}
\end{aligned}
$$

for some $M_{4}>0$ independent of $r$. Similarly, we can get

$$
I_{\left[-\tau_{r}, 0\right]} \leq \sqrt{H} r+M_{4}
$$

Since $I_{[0,1]}$ is independent of $r$, we obtain that

$$
\frac{1}{\sqrt{2}} \int_{-\tau_{r}}^{\tau_{r}} \frac{1}{2}\left|\dot{\gamma}_{r}(t)\right|^{2}+H-V\left(\gamma_{r}(t)\right) d t \leq 2 \sqrt{H} r+M_{3}
$$

for some $M_{3}>0$ independent of $r$. Then by (5.4) and the definition of $b_{r}$, we have

$$
\begin{aligned}
\int_{-T_{r}}^{T_{r}} \sqrt{H-V\left(u_{r}(t)\right)}\left|\dot{u}_{r}(t)\right| d t & \leq\left(\int_{-T_{r}}^{T_{r}} H-V\left(u_{r}(t)\right) d t\right)^{\frac{1}{2}}\left(\int_{-T_{r}}^{T_{r}}\left|\dot{u}_{r}(t)\right|^{2} d t\right)^{\frac{1}{2}} \\
& =\left(2 f\left(q_{r}\right)\right)^{\frac{1}{2}} \\
& \leq\left(2 f\left(\tilde{\gamma}_{r}\right)\right)^{\frac{1}{2}} \\
& \leq \frac{1}{\sqrt{2}} \int_{-\tau_{r}}^{\tau_{r}} \frac{1}{2}\left|\dot{\gamma}_{r}(t)\right|^{2}+H-V\left(\gamma_{r}(t)\right) d t \\
& \leq 2 \sqrt{H} r+M_{3} .
\end{aligned}
$$

Then we finish the proof of this lemma.

By Lemma 4.1, we can see that

$$
M_{5}=\sup _{r>1} \min _{t \in\left(-T_{r}, T_{r}\right)}\left|u_{r}(t)\right| \leq M,
$$

where $M$ is defined in Lemma 4.1. In the following proof, we set a translation as

$$
t^{*}=\inf \left\{t \in\left[-T_{r}, T_{r}\right]|| u_{r}(t) \mid=M_{5}\right\}
$$

and

$$
u_{r}^{*}(t)=u_{r}\left(t^{*}-t\right)
$$

Remark 4 By Lemma 3.2, $u_{r}^{*}$ is also a solution for problem (2.1)-(2.3).

Lemma 5.4 Let $u_{r} \in \Lambda_{r}$ be the solution of problem (2.1)-(2.3) and $u_{r}^{*}$ be defined as (5.6). Then there exists a subsequence $\left\{u_{r_{j}}^{*}\right\}$ of $\left\{u_{r}^{*}\right\}$ convergent to $u_{\infty}$ in $C_{\mathrm{loc}}\left(R, R^{N}\right)$. Furthermore, $u_{\infty}$ is a hyperbolic solution of problem (1.1)-(1.2). 
Proof Step 1: We will show that there exists a subsequence $\left\{u_{r_{j}}^{*}\right\}$ of $\left\{u_{r}^{*}\right\}$ convergent to $u_{\infty}$ in $C_{\text {loc }}\left(R, R^{N}\right)$. By the definition of $u_{r}^{*}$, we can deduce that $u_{r}^{*}$ is a solution of problem (2.1)(2.3). Since $L>M$, we can deduce that $t_{+} \geq t^{*} \geq t_{-}$. Then Lemma 5.2 shows that

$$
-T_{r}+t^{*} \rightarrow-\infty, \quad T_{r}+t^{*} \rightarrow+\infty \quad \text { as } r \rightarrow+\infty
$$

It follows from (2.2) that

$$
\frac{1}{2}\left|\dot{u}_{r}^{*}(t)\right|^{2}+V\left(u_{r}^{*}(t)\right)=H, \quad \forall t \in\left(-T_{r}+t^{*}, T_{r}+t^{*}\right),
$$

which implies that

$$
\left|\dot{u}_{r}^{*}(t)\right|^{2}=2\left(H-V\left(u_{r}^{*}(t)\right)\right), \quad \forall t \in\left(-T_{r}+t^{*}, T_{r}+t^{*}\right) .
$$

Then by (5.3) we have

$$
\left|\dot{u}_{r}^{*}(t)\right| \leq \sqrt{2\left(H+M_{2}\right)} \quad \text { for all } t \in\left(-T_{r}+t^{*}, T_{r}+t^{*}\right),
$$

which implies that

$$
\left|u_{r}^{*}\left(t_{1}\right)-u_{r}^{*}\left(t_{2}\right)\right| \leq\left|\int_{t_{2}}^{t_{1}} \dot{u}_{r}^{*}(s) d s\right| \leq \int_{t_{2}}^{t_{1}}\left|\dot{u}_{r}^{*}(s)\right| d s \leq \sqrt{2\left(H+M_{2}\right)}\left|t_{1}-t_{2}\right|
$$

for each $r>1$ and $t_{1}, t_{2} \in\left[-T_{r}+t^{*}, T_{r}+t^{*}\right]$, which shows that $\left\{u_{r}^{*}\right\}$ is equicontinuous.

Subsequently, we show that $u_{r}^{*}$ is uniformly bounded on any compact set of $R$. Take $a, b \in R$ such that $a<b$. When $r$ is large enough, by Lemma 5.2 , we can see that $[a, b] \subseteq$ $\left[-T_{r}+t^{*}, T_{r}+t^{*}\right]$. Then, for any $t \in[a, b]$, it follows from (5.8) and (5.5) that

$$
\begin{aligned}
\left|u_{r}^{*}(t)\right| & =\left|\int_{0}^{t} \dot{u}_{r}^{*}(t) d t+u_{r}^{*}(0)\right| \\
& \leq\left|\int_{0}^{t} \dot{u}_{r}^{*}(t) d t\right|+\left|u_{r}^{*}(0)\right| \\
& \leq\left|\int_{0}^{t}\right| \dot{u}_{r}^{*}(t)|d t|+\left|u_{r}\left(t^{*}\right)\right| \\
& \leq \sqrt{2\left(H+M_{2}\right)}|t|+M \\
& \leq \sqrt{2\left(H+M_{2}\right)}(|a|+|b|)+M,
\end{aligned}
$$

which implies that

$$
\max _{t \in[a, b]}\left|u_{r}^{*}(t)\right| \leq \sqrt{2\left(H+M_{2}\right)}(|a|+|b|)+M .
$$

Then we have shown that $u_{r}^{*}$ is uniformly bounded on any compact set of $R$ and uniformly equi-continuous on $R$. By the Arzelà-Ascoli theorem, it follows from inequalities (5.9) and (5.10) that there is a subsequence $\left\{u_{r_{j}}^{*}\right\}_{j>0}$ converging to $u_{\infty}$ in $C_{\text {loc }}\left(R, R^{N}\right)$. 
Step 2: We will show that $u_{\infty}$ is a solution of problem (1.1)-(1.2). By Lemma 3.5 and the definition of $u_{r_{j}}^{*}$, we have

$$
\ddot{u}_{r_{j}}^{*}(t)+\nabla V\left(u_{r_{j}}^{*}(t)\right)=0
$$

with

$$
\frac{1}{2}\left|\dot{u}_{r_{j}}^{*}(t)\right|^{2}+V\left(u_{r_{j}}^{*}(t)\right)=H
$$

for each $j>0$ and $t \in\left(-T_{r}+t^{*}, T_{r}+t^{*}\right)$. Take $a, b \in R$ such that $a<b$. By Lemma 4.1, $u_{r_{j}}^{*}$ has no collision uniformly on $[a, b]$. So $\ddot{u}_{r_{j}}(t)$ is continuous on $[a, b]$ and $\ddot{u}_{r_{j}}(t) \rightarrow-\nabla V\left(t, u_{\infty}(t)\right)$ uniformly on $[a, b]$. It follows that $\ddot{u}_{r_{j}}$ is a classical derivative of $\dot{u}_{r_{j}}$ in $(a, b)$ for each $j>0$. Moreover, since $\dot{u}_{r_{j}} \rightarrow \dot{u}_{\infty}$ uniformly on $[a, b]$, we get

$$
\ddot{u}_{\infty}(t)+\nabla V\left(u_{\infty}(t)\right)=0
$$

with

$$
\frac{1}{2}\left|\dot{u}_{\infty}(t)\right|^{2}+V\left(u_{\infty}(t)\right)=H
$$

for all $t \in[a, b]$. Since $a$ and $b$ are arbitrary, we conclude that $u_{\infty}$ satisfies (1.1)-(1.2).

Step 3: We need to show the hyperbolicity of $u_{\infty}(t)$. We prove this conclusion in an indirect way. First, we show that $\left|u_{\infty}(t)\right| \rightarrow+\infty$ as $t \rightarrow+\infty$. Otherwise, there exists a sequence denoted by $t_{n}$ such that $t_{n} \rightarrow+\infty$ as $n \rightarrow+\infty$ and

$$
\left|u_{\infty}\left(t_{n}\right)\right| \leq M_{\infty} \quad \text { for all } n \in N^{+}
$$

for some $M_{\infty}>0$. By (5.1) and (5.2), we obtain that

$$
\int_{-T_{r}+t^{*}}^{T_{r}+t^{*}} \sqrt{H-V\left(u_{r}^{*}(t)\right)}\left|\dot{u}_{r}^{*}(t)\right| d t \geq \int_{t^{*}+t_{-}}^{t^{*}+t_{+}} \sqrt{H-V\left(u_{r}^{*}(t)\right)}\left|\dot{u}_{r}^{*}(t)\right| d t+2 \sqrt{H}(r-L) .
$$

Lemma 5.3 shows that

$$
2 \sqrt{H} r+M_{3} \geq \int_{t^{*}+t_{-}}^{t^{*}+t_{+}} \sqrt{H-V\left(u_{r}^{*}(t)\right)}\left|\dot{u}_{r}^{*}(t)\right| d t+2 \sqrt{H}(r-L) .
$$

Together with (5.7), we have

$$
\begin{aligned}
2 \sqrt{H} L+M_{3} & \geq \int_{t^{*}+t_{-}}^{t^{*}+t_{+}} \sqrt{H-V\left(u_{r}^{*}(t)\right)}\left|\dot{u}_{r}^{*}(t)\right| d t \\
& =\sqrt{2} \int_{t^{*}+t_{-}}^{t^{*}+t_{+}}\left(H-V\left(u_{r}^{*}(t)\right)\right) d t \\
& \geq \sqrt{2} H\left(t_{+}-t_{-}\right) .
\end{aligned}
$$

Subsequently, let $L>\max \left\{M, M_{\infty}, \sigma_{1}\right\}$ be a constant independent of $r$ in the proof of Lemma 5.2. It follows from (5.11) that $t_{-}<+\infty$. But

$$
t_{+} \rightarrow+\infty \quad \text { as } r \rightarrow+\infty \text {, }
$$


which implies that

$$
t_{+}-t_{-} \rightarrow+\infty \quad \text { as } r \rightarrow+\infty
$$

This contradicts (5.12). Then we obtain that $\left|u_{\infty}(t)\right| \rightarrow+\infty$ as $t \rightarrow+\infty$. The proof for $t \rightarrow-\infty$ is similar. Then we finish the proof of Theorem 1 .

\section{Proof of Theorem 2}

Since condition $\left(V_{5}\right)$ is stronger than $\left(V_{3}\right)$, we can obtain the existence of hyperbolic orbits similar to the proof of Theorem 1. Subsequently, we give the estimate of the asymptotic directions of hyperbolic orbits at infinity. Similar to Felmer and Tanaka [9], we set

$$
A(t)=\sqrt{\left|u_{r}(t)\right|^{2}\left|\dot{u}_{r}(t)\right|^{2}-\left(u_{r}(t), \dot{u}_{r}(t)\right)^{2}} \quad \text { and } \quad \omega(t)=\frac{A(t)}{\left|u_{r}(t)\right|\left|\dot{u}_{r}(t)\right|}
$$

Using the motion and energy equations, we have

$$
|\dot{A}(t)| \leq\left|u_{r}(t)\right|\left|\nabla V\left(u_{r}(t)\right)\right|
$$

and

$$
\frac{d \omega}{d t}=\frac{2}{\left|u_{r}(t)\right|\left|\dot{u}_{r}(t)\right|}\left(-\omega \sqrt{1-\omega^{2}} \operatorname{sign}\left(u_{r}(t), \dot{u}_{r}(t)\right)\left(H-V\left(u_{r}(t)\right)\right)+\left|u_{r}(t)\right|\left|\nabla V\left(u_{r}(t)\right)\right|\right) .
$$

Lemma 6.1 (see[9]) Assume that $u_{r}$ is a solution for problem (2.1)-(2.3) obtained in Lemma 3.5. For any $\eta \in(0,1)$, there exists $L_{\eta} \geq m$ such that if

$$
\left|u_{r}\left(t_{0}\right)\right| \geq L_{\eta}, \quad\left(u_{r}\left(t_{0}\right), \dot{u}_{r}\left(t_{0}\right)\right)>0 \quad \text { and } \quad \omega\left(t_{0}\right)<\eta
$$

for some $t_{0} \in\left(-T_{r}, T_{r}\right)$, then we have for $t \in\left[t_{0}, T_{r}\right]$

(i) $\omega(t)<\eta$,

(ii) $\frac{d}{d t}\left|u_{r}(t)\right| \geq \sqrt{1-\eta^{2}}\left|\dot{u}_{r}(t)\right|$,

(iii) $\frac{d}{d t}\left|u_{r}(t)\right| \geq \sqrt{2\left(1-\eta^{2}\right) H}$,

(iv) $\left|u_{r}(t)\right| \geq\left|u_{r}\left(t_{0}\right)\right|+\sqrt{2\left(1-\eta^{2}\right) H}\left(t-t_{0}\right)$.

Lemma 6.2 Let $u_{r}$ be a solution for problem (2.1)-(2.3) obtained in Lemma 3.5 satisfying (6.1) and $\left|u_{r}(t)\right| \geq \sigma_{2}$ with $t \geq t_{0}$ for certain $t_{0} \in\left(-T_{r}, T_{r}\right)$ with $\eta \in\left(0, \frac{1}{2}\right)$ and $L_{\eta}$ be as in Lemma 6.1. Then, for $t \geq t_{0}$, there exist $M_{6}, M_{7}>0$ independent of $\eta, u_{r}(t)$ and $t_{0}$ such that

$$
\left|\frac{u_{r}(t)}{\left|u_{r}(t)\right|}-\frac{u_{r}\left(t_{0}\right)}{\left|u_{r}\left(t_{0}\right)\right|}\right| \leq M_{6} \eta+\frac{M_{7}}{\left|u_{r}\left(t_{0}\right)\right|^{\beta}}
$$

Proof By Lemma 5.1, (iii) of Lemma 6.1 and $\left(\mathrm{V}_{5}\right)$, we can estimate $A(t)$ as follows.

$$
\begin{aligned}
A(t) & \leq A\left(t_{0}\right)+\int_{t_{0}}^{t}\left|u_{r}(s)\right|\left|\nabla V\left(u_{r}(s)\right)\right| d s \\
& \leq A\left(t_{0}\right)+\frac{M_{7}}{\sqrt{2\left(1-\eta^{2}\right) H}} \int_{t_{0}}^{t}\left|u_{r}(s)\right|\left|\nabla V\left(u_{r}(s)\right)\right| \frac{d}{d s}\left|u_{r}(s)\right| d s
\end{aligned}
$$




$$
\begin{aligned}
& \leq A\left(t_{0}\right)+\frac{M_{7}}{\sqrt{2\left(1-\eta^{2}\right) H}} \int_{\left|u_{r}\left(t_{0}\right)\right|}^{\left|u_{r}(t)\right|} \varphi\left|\nabla V\left(\varphi \frac{u_{r}(s)}{\left|u_{r}(s)\right|}\right)\right| d \varphi \\
& \leq A\left(t_{0}\right)+\frac{M_{7} \rho_{0}}{\sqrt{2\left(1-\eta^{2}\right) H}} \int_{\left|u_{r}\left(t_{0}\right)\right|}^{\left|u_{r}(t)\right|} \frac{1}{\varphi^{\kappa-1}} d \varphi \\
& \leq A\left(t_{0}\right)+\frac{M_{7} \rho_{0}}{\sqrt{2\left(1-\eta^{2}\right) H}(\beta-1)} \frac{1}{\left|u_{r}\left(t_{0}\right)\right|^{\kappa-2}} \\
& \leq A\left(t_{0}\right)+\frac{M_{8}}{\left|u_{r}\left(t_{0}\right)\right|^{\kappa-2}}
\end{aligned}
$$

for some $M_{8}>0$ independent of $r$. Since we have

$$
\left|\frac{d}{d t} \frac{u_{r}(t)}{\left|u_{r}(t)\right|}\right|=\frac{A(t)}{\left|u_{r}(t)\right|^{2}}
$$

then it follows from (iii) of Lemma 6.1, (6.2) and (6.3) that

$$
\begin{aligned}
\left|\frac{u_{r}(t)}{\left|u_{r}(t)\right|}-\frac{u_{r}\left(t_{0}\right)}{\left|u_{r}\left(t_{0}\right)\right|}\right| & \leq \int_{t_{0}}^{t} \frac{A(s)}{\left|u_{r}(s)\right|^{2}} d s \\
& \leq\left(A\left(t_{0}\right)+\frac{M_{8}}{\left|u_{r}\left(t_{0}\right)\right|^{\kappa-2}}\right) \int_{t_{0}}^{t} \frac{1}{\left|u_{r}(s)\right|^{2}} d s \\
& \leq\left(A\left(t_{0}\right)+\frac{M_{8}}{\left|u_{r}\left(t_{0}\right)\right|^{\kappa-2}}\right) \frac{1}{\sqrt{2\left(1-\eta^{2}\right) H}} \int_{t_{0}}^{t} \frac{1}{\left|u_{r}(s)\right|^{2}} \frac{d}{d s}\left|u_{r}(s)\right| d s \\
& \leq\left(A\left(t_{0}\right)+\frac{M_{8}}{\left|u_{r}\left(t_{0}\right)\right|^{\kappa-2}}\right) \frac{1}{\sqrt{2\left(1-\eta^{2}\right) H}} \frac{1}{\left|u_{r}\left(t_{0}\right)\right|} .
\end{aligned}
$$

By energy equation and the definition of $t_{0}$, we have

$$
A\left(t_{0}\right)=\omega\left(t_{0}\right)\left|u_{r}\left(t_{0}\right)\right|\left|\dot{u}_{r}\left(t_{0}\right)\right| \leq \eta\left|u_{r}\left(t_{0}\right)\right| \sqrt{2\left(H-V\left(u_{r}\left(t_{0}\right)\right)\right)},
$$

which implies that for some $M_{6}, M_{7}>0$ independent of $r$

$$
\left|\frac{u_{r}(t)}{\left|u_{r}(t)\right|}-\frac{u_{r}\left(t_{0}\right)}{\left|u_{r}\left(t_{0}\right)\right|}\right| \leq M_{6} \eta+\frac{M_{7}}{\left|u_{r}\left(t_{0}\right)\right|^{\kappa}}
$$

which proves this lemma.

By Lemmas 5.3, 6.1 and 6.2, similar to [9], we have the following lemma.

Lemma 6.3 (see [9]) For any $\varepsilon>0$, there exists $M_{9}>0$ such that for $r>M_{9}$

$$
u_{r}\left(\left[t^{*}, T_{r}\right]\right) \bigcap\left\{|x| \geq M_{9}\right\} \subset\left\{y \in R^{N}:\left|\frac{y}{|y|}-e_{+}\right|<\varepsilon\right\},
$$

where $e_{+}$is the given direction defined in $\Lambda_{r}$ and $t^{*}$ is defined as (5.5).

Let $t_{1} \geq t^{*}$ such that $\left|u_{r}\left(t_{1}\right)\right|=L_{\eta}$. Then, for any $\varepsilon>0$, we get

$$
\left|\frac{u_{r}(t)}{\left|u_{r}(t)\right|}-e_{+}\right|<\varepsilon
$$


for all $t \geq t_{1}$, which implies that

$$
\frac{u_{\infty}(t)}{\left|u_{\infty}(t)\right|} \rightarrow e_{+} \quad \text { as } t \rightarrow+\infty
$$

Similarly, we can get

$$
\frac{u_{\infty}(t)}{\left|u_{\infty}(t)\right|} \rightarrow e_{-} \quad \text { as } t \rightarrow-\infty
$$

From the above discussion, we can see that there exists at least one hyperbolic solution for (1.1)-(1.2) with $H>0$ which has the given asymptotic direction at infinity. Then we finish the proof of Theorem 2 .

\section{Competing interests}

The authors declare that they have no competing interests.

\section{Authors' contributions}

All the authors contributed equally and significantly in writing this article. All the authors read and approved the final manuscript.

\section{Acknowledgements}

The authors are very grateful to the referees for their very helpful comments and suggestions, which greatly improved the presentation of this paper. This work is supported by the National Natural Science Foundation of China (No. 11301358).

Received: 20 March 2015 Accepted: 13 May 2015 Published online: 30 May 2015

\section{References}

1. Ambrosetti, A: Critical points and nonlinear variational problems. Mém. Soc. Math. France 49, 1-139 (1992)

2. Ambrosetti, A, Coti Zelati, V: Closed orbits of fixed energy for singular Hamiltonian systems. Arch. Ration. Mech. Anal. $112,339-362(1990)$

3. Ambrosetti, A, Coti Zelati, V: Closed orbits of fixed energy for a class of N-body problems. Ann. Inst. Henri Poincaré, Anal. Non Linéaire 9, 187-200 (1992)

4. Barutello, V, Terracini, S, Verzini, G: Entire parabolic trajectories as minimal phase transitions. Calc. Var. Partial Differ. Equ. 49, 391-429 (2014)

5. Bahri, A, Rabinowitz, PH: A minimax method for a class of Hamiltonian systems with singular potentials. J. Funct. Anal. 82, 412-428 (1989)

6. Cerami, G: Un criterio di esistenza per i punti critici su varietà illimitate. Rend. Acad. Sci. Lett. Ist. Lombardo 112 , 332-336 (1978)

7. Chang, K-C: Infinite Dimensional Morse Theory and Multiple Solution Problems. Birkhäuser, Basel (1993)

8. Degiovanni, M, Giannoni, F: Periodic solutions of dynamical systems with Newtonian type potentials. Ann. Sc. Norm. Super. Pisa, Cl. Sci. 15, 467-494 (1988)

9. Felmer, P, Tanaka, K: Hyperbolic-like solutions for singular Hamiltonian systems. Nonlinear Differ. Equ. Appl. 7, 43-65 (2000)

10. Gordon, W: Conservative dynamical systems involving strong forces. Trans. Am. Math. Soc. 204, 113-135 (1975)

11. LV, Y, Tang, C-L: Existence of even homoclinic orbits for second-order Hamiltonian systems. Nonlinear Anal. 67(7), 2189-2198 (2007)

12. Mawhin, J, Willem, M: Critical Point Theory and Hamiltonian Systems. Appl. Math. Sci., vol. 74. Springer, New York (1989)

13. Verhulst, F: Nonlinear Differential Equations and Dynamical Systems. Springer, Berlin (1990)

14. Rabinowitz, PH: Homoclinic orbits for a class of Hamiltonian systems. Proc. R. Soc. Edinb., Sect. A 114, 33-38 (1990)

15. Rabinowitz, PH: Periodic and heteroclinic orbits for a periodic Hamiltonian system. Ann. Inst. Henri Poincaré, Anal. Non Linéaire 6(5), 331-346 (1989)

16. Serra, E, Terracini, S: Noncollision solutions to some singular minimization problems with Keplerian-like potentials. Nonlinear Anal. TMA 22, 45-62 (1994)

17. Wu, D-L, Wu, X-P, Tang, C-L: Homoclinic solutions for a class of nonperiodic and noneven second-order Hamiltonian systems. J. Math. Anal. Appl. 367, 154-166 (2010)

18. Wu, D-L, Zhang, SQ: Homoclinic orbits to infinity for second order Hamiltonian systems with fixed energy. (submitted)

19. Maderna, E, Venturelli, A: Globally minimizing parabolic motions in the Newtonian N-body problem. Arch. Ration. Mech. Anal. 194, 283-313 (2009) 